\title{
Perforation of the bowel from intrauterine contraceptive devices: case series and discussion
}

Received: 30 December 2005 / Accepted: 10 March 2006 / Published online: 7 July 2006

(C) Springer-Verlag Berlin / Heidelberg 2006

\begin{abstract}
The intrauterine contraceptive device is used extensively in the female population. Serious complications are rare, but they do occur. We discuss three cases of bowel perforation caused by these devices following their perforation through the uterine wall. It is important that cases of perforation be recognised swiftly and the possibility of involvement of other organs considered.
\end{abstract}

Keywords Intrauterine contraceptive device -

Complications $\cdot$ Bowel perforation

\section{Introduction}

In $2003 / 2004$, half $(52 \%)$ of women aged 16-49 in the United Kingdom were using at least one nonsurgical method of contraception [1]. The intrauterine contraceptive device (IUCD) was used by $4 \%$ of these women. In this case series we discuss three cases of bowel perforation following insertion of various IUCDs and their clinical outcomes.

C. Oxby

Leeds General Infirmary,

Leeds, UK

S. Jones

Bradford Royal Infirmary,

Bradford, UK

S. Acharya

Airedale General Hospital,

Keighley, UK

C. Oxby $(\bowtie)$

Department of Obstetrics, Clarendon Wing,

Leeds General Infirmary,

Belmont Grove,

Leeds, LS2 9NS, UK

e-mail: claireoxby@hotmail.com

Tel.: +44-113-2432799

\section{Case one}

A 28-year-old woman presented to her general practitioner (GP) for removal of her IUCD because she wished to conceive. Five years previously she had had a Novagard IUCD inserted for contraception by her GP, and she had undergone four satisfactory coil checks on a yearly basis. She had no significant past medical history and had two children by normal vaginal delivery and no other pregnancies. At presentation, coil threads where seen on examination, but during attempted removal the string snapped off, with the tip of the device visible at the external cervical os. Further attempts to remove the device with a long-handled clip proved unsuccessful. The woman was referred to the outpatient hysteroscopy clinic for IUCD removal.

Hysteroscopic examination revealed that the uterine cavity was empty, and an ultrasound scan of the pelvis located the IUCD lying just outside the uterus. It is possible that the IUCD perforated the uterus during the initial removal attempt or during hysteroscopy, but there was no evidence of trauma, making this unlikely. At laparoscopy there were multiple omental adhesions covering the uterus, especially on the left side, and no evidence of the coil. Therefore, a laparotomy was undertaken and the general surgeons asked to attend. The coil was found deeply embedded in the left broad ligament with the sigmoid colon densely adherent over the top of it and to the uterus. The coil was removed, and a fistulous track was found going into the serosa of the large bowel with sparing of the mucosa. This area was oversewn, and an omental patch was placed on the surface. The patient made a good postoperative recovery with no bowel complications.

\section{Case two}

A 25-year-old woman presented to her GP with a positive pregnancy test. She had had two children in the past by normal vaginal delivery and no other pregnancies. There was no past medical history of note. This woman had a 
Nova-T IUCD in situ, which had been inserted 4 months previously by her GP; there was no history of difficulty at insertion. An ultrasound scan of the pelvis at the time of presentation showed a 6 -week singleton viable intrauterine pregnancy, with the IUCD lying to the left of the uterus in the pouch of Douglas. Conservative management was employed, and the women went on to have an uncomplicated pregnancy and normal vaginal delivery at term. No IUCD was identified at delivery. Six weeks after delivery, a repeat ultrasound scan was performed, and the coil was not seen in the uterus. The patient underwent laparoscopy, which found the coil embedded in the lumen of the sigmoid colon. General surgeons were asked to review, and the coil was removed and the bowel oversewn. In the postoperative period the patient made a good recovery with no complications.

\section{Case three}

A 31-year-old woman presented to her GP with pelvic pain. Five days earlier she had had an IUCD inserted at a local family-planning clinic. Insertion was associated with severe pain, which had not resolved. This woman had had five children in the past by normal vaginal delivery and no other pregnancies, and the last child was over 1 year old. At presentation to her GP, this woman was examined, and the threads from the IUCD could not be demonstrated. Her GP arranged for an ultrasound scan, which reported the IUCD to be outside the endometrial cavity on the right side of the uterus. It appeared to extend into the region of the right tube with its tip near the right ovary. There was a small amount of fluid in the pouch of Douglas. A referral to the outpatient department was made, and the woman was seen 6 months later; the reason for the delay was the patient's cancellation of her initial appointment. Once she had been seen in the outpatient department, arrangements were made for a laparoscopy and possible laparotomy. At laparoscopy the IUCD was identified in the right broad ligament. This was divided, revealing adhesions around the end of the IUCD, with its body lying in the lumen of the small bowel near the terminal ileum. The terminal ileum itself was adherent to the sigmoid colon. The general surgeons were asked to review, and a laparotomy was undertaken to remove the IUCD. The subsequent defects in the small bowel were oversewn. The woman made an uneventful postoperative recovery.

\section{Discussion}

IUCDs have been used throughout the world since the 1960s. Their use is increasing, especially since the introduction of hormone-releasing devices broadening the therapeutic range. The effectiveness of any method of contraception is measured by the number of unwanted pregnancies that occur during 100 woman-years of exposure, i.e. during 1 year of use by 100 women who are normally fertile and having regular coitus. This is known as the "Pearl index." Both the newer copper-containing devices and the levonorgestrel-releasing device are effective methods of contraception, with a Pearl index of less than 1 per 100 woman-years [2].

Potential side effects of the IUCD include expulsion, menstrual disturbances, ectopic pregnancy, pelvic infection, and perforation. Perforation is rare but, as highlighted by these cases, can have serious consequences. The rate of perforation quoted by the Faculty of Family Planning and Reproductive Health Care (FFPRHC) Clinical Effectiveness Unit is less than one in 1,000 insertions [3, 4]. It is advised that women should be offered instruction on how to check for the IUCD and its threads and be told that if they are unable to feel them, the device may have been expelled. Expulsion is the most common cause of IUCD failure, with a risk of around 1 in 20, frequently in the first 3 months [5]. Alternative contraception should then be used until medical advice has been obtained $[3,4]$.

Perforation usually occurs at the time of insertion but may be asymptomatic and hence go unnoticed. It has also been hypothesised that an IUCD that is embedded in the uterus can later be forced through the wall by uterine contractions [6]. Diagnosis is made by the lack of visible threads at the cervix and evidence of the IUCD outside the uterus on either ultrasound scan or plain abdominal x-ray. When perforation is suspected, an ultrasound scan of the pelvis should be undertaken initially. If this shows no evidence of the IUCD, an abdominal x-ray should be done. It is not safe practice to assume that IUCD has been expelled just because an ultrasound scan is negative.

Traditionally, perforation of another lumen, such as the bowel, by an IUCD cannot be diagnosed without more invasive techniques such as laparoscopy. Recent advances, however, have looked at the role of computed tomography in diagnosing IUCD placement [7]. Most IUCDs within the abdominal cavity do not perforate other viscera but do cause adhesions. It has been questioned whether their surgical removal is necessary [8]; however, this case series illustrates the harm IUCDs can do if left in the intraabdominal cavity. Other single case reports [9-13] also support surgical removal, as do recommendations from the FFPRHC [3].

\section{Conclusion}

IUCDs are an effective method of contraception, but care is needed at their insertion, and continued follow-up is required to identify cases of perforation. It is essential that medical personnel be adequately trained in the fitting and management of IUCDs.

\section{References}

1. Dawe F, Rainford L (2004) Contraception and sexual health 2003. National Statistics, London, UK:HMSO 1-53. http:// www.statistics.gov.uk 
2. French RS, Cowan FM, Mansour DJA et al (2000) Implantable contraceptives (subdermal implants and hormonally impregnated intrauterine systems) verses other forms of reversible contraceptives: two systematic reviews to assess relative effectiveness, acceptability, tolerability and cost effectiveness (review). Health Technol Assess 4:i-vi, 1-107

3. Penney G, Brechin S, de Souza A, Bankowska U, Belfield T, Gormley M, Olliver M, Hampton N, Howlett-Shipley R, Hughes S, Mack N, O'Brien P, Rowlands S, Trewinnard K; Faculty of Family Planning and Reproductive Health Care Clinical Effectiveness Unit (2004) FFPRHC Guidance (January 2004). The copper intrauterine device as long-term contraception. J Fam Plann Reprod Health Care 30(1):29-42

4. Faculty of Family Planning and Reproductive Health Care (2004) FFPRHC Guidance (April 2004). The levonorgestrelreleasing intrauterine system (LHG-IUS) in contraception and reproductive health. J Fam Plann Reprod Health Care 30 (2):99-109

5. World Health Organization (1987) Mechanism of action, safety and efficacy of intrauterine devices. WHO Technical Report, series 753:1-91

6. Carson SA, Gatlin A, Mazur M (1981) Appendiceal perforation by copper-7 intrauterine contraceptive device. Am J Obstet Gynaecol 141(5):586-587
7. El Saadi A, Mohandes I, Emad M, Abdulbaqi H (2004) The role of $\mathrm{CT}$ scan in laparoscopic retrieval of a perforated intrauterine device (IUD). Gynae Surgery 1(4):247-250

8. Markovitch O, Klein Z, Gidoni Y, Holzinger M, Beyth Y (2002) Extrauterine mislocated IUD: is surgical removal mandatory? Contraception 6(2):105-108

9. Nceboz US, Ozcakir HT, Uyar Y, Caglar H (2003) Migration of an intrauterine contraceptive device to the sigmoid colon: a case report. Eur J Contracept Health Care 8(4):229-232

10. Disu S, Boret A (2004) Asymptomatic ileal perforation of an intrauterine device. Arch Gynecol Obstet 269(3):230-231

11. Chen CP, Hsu TC, Wang W (1998) Ileal penetration by a Multiload-Cu 375 intrauterine contraceptive device. A case report with a review of literature. Contraception 58:295-304; erratum in 58 :following 389

12. Gronlund B, Blaabjerj J (1991) Serious intestinal complication five years after insertion of a Nova-T. Contraception 44 (5):517-520

13. Pirwany IR, Boddy K (1997) Colocolic fistula caused by a previously inserted intrauterine device. Case report. Contraception 56(5):337-339 